\title{
O ESTUDO DO TRATAMENTO DA INFORMAÇÃO NOS LIVROS DIDÁTICOS DAS SÉRIES INICIAIS DO ENSINO FUNDAMENTAL ${ }^{*}$
}

\section{A study of the usage of information in text books from the beginning grades of elementary teaching}

Maria Patrícia Freitas de Lemos ${ }^{1}$

\begin{abstract}
Resumo: 0 presente estudo investiga o tratamento da informação expresso em livros didáticos brasileiros apresentados em três coleções analisadas pelo PNLD (2000 e 2004) que foram recomendadas com distinção das séries iniciais do ensino fundamental. As atividades foram analisadas a partir dos tipos de gráficos e conteúdos matemáticos abordados nas tarefas analisadas. Considerando o importante papel que os livros didáticos assumem na educação brasileira esse estudo aponta-nos que 0 trabalho com tratamento da informação, realizado pelos livros didáticos, encontra-se distante de possibilitar aos alunos a construção de procedimentos para coletar, organizar, comunicar e interpretar dados. $\mathrm{O}$ tratamento da informação, na maioria das coleções analisadas, não é explorado no decorrer de todos os livros didáticos, aparecendo apenas no capítulo de estatística e probabilidade.
\end{abstract}

Palavras-chave: interpretação de gráficos. educação matemática. livro didático.

Abstract: The present research deals with the use of information expressed in Brazilian text books in three collections analized by PN L D ( N ational Plan of Didatic Book ) ( 2000 and 2004 ) which were reommended with distinction for the beginning grades of elementary teaching. The activities were analyzed for the type of graphics and maths contents. C onsidering the important role that text books play on Brazilian education, this study shows us that the work with the use of information within tex t books is not giving students the constructive prooedures needed to collect, organize, communicate and interpret information. There is little use of information in the majority of the analyzed ollections, appearing only in the statistics and probability chapters.

Keywords: graphic interpretation. math education. didactic book.

\footnotetext{
* E ste artigo faz parte de um estudo maior sobre as abordagens dadas ao tratamento da informação nos livros didáticos recomendados com distinção e recomendados pelo PN L D 2004, que encontra-se em andamento.

${ }^{2}$ Professora, D epartamento de Ciências Sociais, E ducação e D esporto, Campus Ministro Reis V elloso, U niversidade Federal do Piauí. E-mail: mpflemos@ ufpi.br
} 


\section{Introdução}

A tualmente, a sociedade tem atribuído grande importância às informações transmitidas pelos meios de comunicação. Essas, na maioria das vezes, vêm expressas por listas, tabelas e gráficos de vários tipos.

Particularmente, a mídia impressa utiliza gráficos para ilustrar seus argumentos jornalísticos. Nesse sentido, é preciso compreender que o gráfico, no contexto da mídia impressa, está diretamente vinculado a determinadas intenções de quem estrutura a matéria. Nesse aspecto, é importante que tenhamos os conhecimentos necessários para entendermos o significado desses dados e, ao mesmo tempo, que saibamos interpretar os diferentes instrumentos que são utilizados para representá-los. Por outro lado, para desenvolver a capacidade de entender 0 argumento apresentado e, também, de criticá-lo, é importante saber selecionar, organizar e entender essas informações mostradas a todo momento pela mídia.

Alguns estudos têm apontado que a compreensão de gráficos não depende, apenas, de conhecimentos formais, mas, também, pode ser influenciada por fatores, tais como as próprias expectativas dos sujeitos, como verificaram Carraher et al. (1995) e Meira (1998).

Nas últimas décadas, a importância da compreensão e do ensino, desde cedo, desse sistema de representação nas escolas tem sido reconhecida internacionalmente, por diversos documentos relativos ao currículo da Matemática (National Council of Teachers of Mathematics - NCTM, Psychology of Mathematics Education - PME, International Conferences on Mathematics Education - ICME, entre outros). No Brasil, desde 1997 os Parâmetros Curriculares Nacionais - PCN - vêem defendendo o trabalho com este conteúdo desde as séries iniciais.

Esse documento propõe que seja realizado um trabalho efetivo com as séries iniciais, envolvendo o ensino de interpretação e construção de gráficos e tabelas. Segundo os PCNs, o ensino de gráficos deve possibilitar o encontro entre as experiências diárias, matemáticas complexas e idéias científicas, além de se mostrar eficaz para investigar a ocorrência simultânea de matemática presente na vida cotidiana e dos conceitos matemáticos formais. "A finalidade não éa de que os alunos aprendam apenas a ler e a interpretar representa ọ̃es gráficas, mas que se tornem capazes de descrever e interpretar sua realidade, usando conhecimentos matemátios" (p. 69). Sendo assim, a escola tem sido solicitada a colaborar com o ensino do tratamento da informação em sala de aula.

Diante disso, observamos que recai sobre o professor, principalmente, o papel de ensinar esses conteúdos aos alunos. E, como sabemos, um dos principais recursos de ensino utilizados pelo professor é 0 livro didático, recurso que se configura numa das poucas formas de documentação e de consulta usada por professores e alunos nas escolas públicas (Brasil, 2004).

Segundo Silva (1996) a escola concebe o livro (didático ou não) como um instrumento básico, um complemento primeiro das funções pedagógicas exercidas pelo professor. Lajolo (1996) reafirma esta concepção e acrescenta que os livros didáticos não devem ser os únicos 
materiais de que os professores e alunos irão valer-se no processo de ensino-aprendizagem, mas que pode ter muita influência na qualidade do aprendizado resultante das atividades escolares, especialmente em nossa sociedade, uma vez que no decorrer de sua utilização os livros acabaram determinando conteúdos, condicionando estratégias de ensino e marcando de forma bastante incisiva o que se ensina e como se ensina em nossas escolas.

Logo, segundo Albuquerque (2002), questionar os livros didáticos é questionar 0 próprio ensino que neles está cristalizado.

Os livros didáticos voltaram a ser alvo das atenções desde que o Ministério da Educação passou a subordinar a compra desses materiais didáticos a uma análise oficial prévia efetuada por especialistas. Esse programa, conhecido como Programa Nacional do Livro Didático - PNLD (Brasil, 2004), tem por objetivos básicos a aquisição e distribuição, universal e gratuita, de livros didáticos para os alunos das escolas públicas do Ensino Fundamental brasileiro. A fim de assegurar a qualidade dos livros a serem adquiridos nas escolas públicas, 0 PNLD desenvolve um processo de avaliação pedagógica das obras nele inscritas, executado pela Secretaria da Educação Fundamental (SEF), do Ministério da Educação. A partir desse trabalho, foi criado um Guia de Livros Didáticos, no qual todos os livros que reúnem qualidades suficientes para serem recomendados (om ou sem ressalvas) são apresentados aos professores para que possam escolher aqueles que julguem mais apropriados a seus propósitos.

Com base na análise do PNLD, mediante o Guia de Livros Didáticos, selecionamos para este trabalho as coleções de $1^{\underline{a}}$ a $4^{\underline{a}}$ séries do Ensino Fundamental que receberam a menção "recomendado com distinção". Foram elas: mato, (2003);

Coleção: Matemática com Sarquis, Autor: Eduardo Sarquis Soares, Editora: For-

Coleção: Novo Tempo, Autores: Luis Márcio Pereira Imenes, Marcelo Cestari Terra Lellis e José Jakubovic, Editora: Scipione, (2003) Ática, (2003).

Coleção: Vivência e Construção - Matemática, Autor: Luiz Roberto D ante, Editora:

A análise dos livros didáticos permitiu verificar se os mesmos propõem atividades com tratamento da informação e, em caso afirmativo, quais os tipos de gráficos abordados em cada atividade.

\section{Representação gráfica de dados estatísticos}

A apresentação gráfica constitui complemento importante da representação de dados estatísticos por meio de tabelas. Esse recurso permite ter uma visualização imediata da distribuição dos valores observados, ao contrário da apresentação tabular.

Apesar de as tabelas apresentarem uma síntese dos dados, na maior parte das vezes elas não retêm a atenção do observador, daí a Estatística se valer de outro recurso: os gráficos. Em linhas gerais, eles propiciam uma idéia preliminar mais satisfatória da concentração e dispersão dos valores, uma vez que, por eles, os dados estatísticos se apresentam, em termos de grandezas, visualmente interpretáveis (Toledo, 1985). Em outras palavras, a representação gráfica permite uma visualização global do fenômeno em estudo, possibilitando uma análise rápida e segura. 
No entanto, é de fundamental importância que ele seja feito de forma criteriosa e seguindo certas características, como simplicidade, clareza e veracidade, evitando, assim, que sua interpretação deixe dúvidas ao leitor (Silva, 1998).

\section{Classificação dos gráficos estatísticos}

De acordo com a forma, os gráficos podem ser classificados como:

- Diagramas - gráficos geométricos dispostos em duas dimensões. São os gráficos mais usados na representação de séries estatísticas e se apresentam em uma grande variedade de tipos.

- Cartogramas - são ilustrações relativas a cartas geográficas, largamente difundidas em Geografia, História e D emografia.

- Estereogramas - dispostos em três dimensões, representam volumes. Muitas vezes são confeccionados em cartolina ou madeira, quando não desenhados em perspectiva (Toledo, 1985).

Embora possamos distribuir os gráficos estatísticos por esses três tipos distintos, em nosso estudo vamos nos deter, apenas, em alguns tipos de gráficos em diagramas:

- $\mathrm{G}$ ráficos em barras horizontais;

- Gráficos em colunas verticais;

- G ráficos em barras e em colunas compostas;

- G ráficos em barras e em colunas agrupadas;

- G ráficos em barras bidirecionais;

- G ráficos de percentagem complementares.

Nossa escolha pelos gráficos de barras e colunas surgiu em decorrência de serem eles muito utilizados pelos meios de comunicação, como revistas e jornais, entre outros (Lima, 1998), e, também, por se apresentarem como preferidos pelos livros didáticos, de modo geral, o que constatamos quando da análise das coleções Novo Tempo, Matemática com o Sarquis, e Vivência e Construção - Matemática, livros didáticos indicados pelo MEC com distinção.

\section{Metodologia}

Analisamos, quantitativa e qualitativamente, três coleções de livros didáticos de matemática de 1a a 4⿳亠口冋 série do Ensino Fundamental, contabilizando um total de 12 volumes. Essas coleções foram selecionadas por terem recebido "recomendação com distinção".

O processo de análise desenvolveu-se do seguinte modo:

- Primeiramente, selecionamos, em todas as oleẹ̃es, as atividades œm gráficos e observamos cmo elas eram distribuídas no livro didático.

- N 0 segundo momento, contabilizamos os tipos de gráficos apresentados nas atividades de cada oleccão, por volume, ou seja, quais os tipos de gráficos que mais apareceram em cada questão œmo, por ex emplo, gráficos de linha, setores (pizza), barras e colunas, entre outros.

- $\mathrm{N}$ o terceiro momento realizamos uma análise sobre os tipos de conteúdos trabalhados em 
cada atividade, ou seja, que tipos de conteúdo cada questão de ensino e interpretação de gráficos vêm explorando.

O procedimento de coleta dos dados baseou-se numa metodologia qualitativa e quantitativa que foi composta de sete etapas distribuídas da seguinte maneira:

\begin{tabular}{|l|l|}
\hline PASSO & \multicolumn{1}{|c|}{ ATIVIDADE } \\
\hline $1{ }^{0}$ & Escolhas das coleções a partir do PNLD (BRASIL, 2000 e 2004). \\
\hline $2^{\underline{0}}$ & $\begin{array}{l}\text { Seleção de todas as atividades com gráficos contidos nas 3 coleções, } \\
\text { perfazendo um total de 12 volumes. }\end{array}$ \\
\hline $3^{\mathbf{o}}$ & $\begin{array}{l}\text { Contagem de todos os tipos de gráficos existentes em cada atividade, } \\
\text { com tratamento da informação selecionada nos 12 volumes }\end{array}$ \\
\hline $4^{\underline{0}}$ & $\begin{array}{l}\text { Análise dos conteúdos trabalhados/ abordados em cada atividade } \\
\text { selecionada nos 12 volumes. }\end{array}$ \\
\hline 50 & Análise e discussão dos dados. \\
\hline
\end{tabular}

\section{Resultados}

Vale ressaltar que esses resultados equivalem à análise dos livros didáticos indicados pelo PNLD (2000), visto que se encontram em andamento as análises dos livros didáticos avaliados pelo PNLD (2004).

D as três coleções analisadas encontramos 91 questões relacionadas ao tratamento da informação distribuídas no decorrer de todos os livros didáticos analisados. Para melhor compreensão dos dados, chamaremos de coleção 10 livro Matemática com o Sarquis; coleção 2 o livro Vivência e Construção - Matemática e coleção 3 o livro Novo Tempo.

No que se refere à distribuição das atividades com tratamento da informação, em cada coleção observamos que apenas a Matemática com o Sarquis, coleção 1, apresenta 0 trabalho com gráficos no decorrer de todo o livro, ou seja, do início ao final dos volumes, fator não observado nas demais coleções. Além disso, ressaltamos, também, que, apenas essa coleção apresenta o trabalho com gráfico articulado com os outros campos da Matemática. Nas duas últimas, o trabalho com construção e interpretação de gráficos aparece, apenas, no capítulo de estatística e probabilidade.

O utro fator interessante é que, em todas as coleções analisadas, 0 trabalho com 0 tratamento da informação só começa a partir do $2^{\circ}$ volume. Nenhuma das coleções explora 0 ensino de construção e interpretação de gráficos e tabelas no $1^{\circ}$ volume, contrariando, assim, as próprias determinações dos Parâmetros Curriculares Nacionais - PCNs (Brasil, 1997), que vêm defendendo o trabalho com tratamento da informação desde as séries iniciais. 
Com relação às 91 questões sobre tipos de gráficos apresentadas nas coleções, observamos que, desse total, 64 (70\%) estão presentes na coleção 1; 8 (9\%), na coleção 2; e 19 (20\%), na coleção 3. 0 gráfico abaixo representa melhor esses dados:

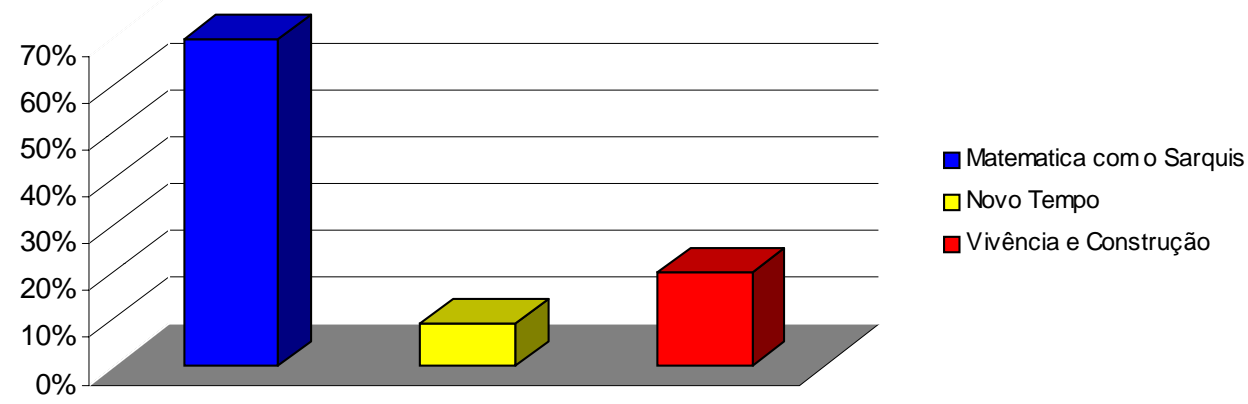

\section{Gráfico 1}

Gráfico de freqüência total dos tipos de gráficos por coleção

Entretanto, apesar de a coleção 1 apresentar a maior quantidade de tipos de gráficos, quando analisamos a distribuição do percentual das questões que acompanham as atividades com gráficos verificamos que esta coleção aparece em segundo lugar em relação à quantidade de questões presentes nas atividades com interpretação e construção de gráficos. Esse resultado refere-se a todos os volumes, como pode ser observado no gráfico abaixo:

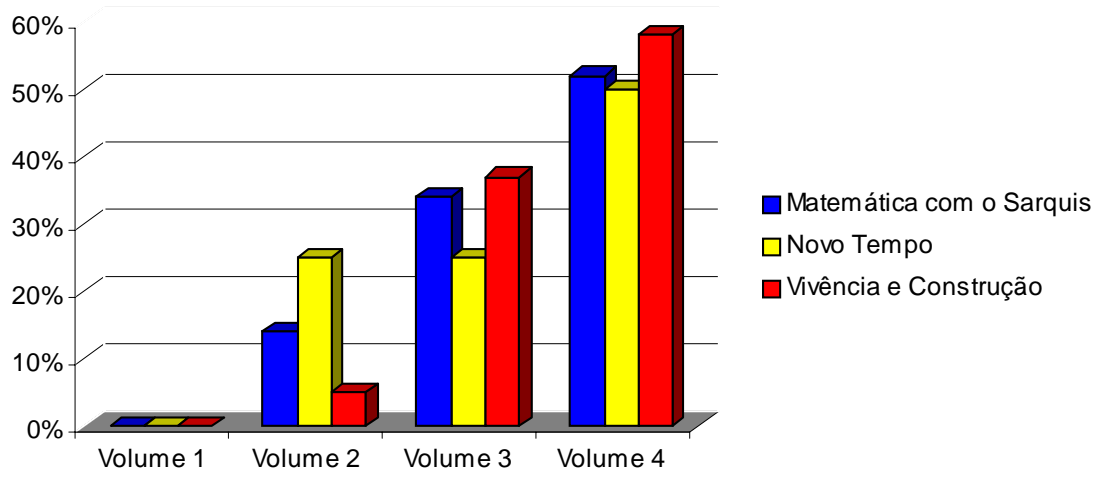

\section{Gráfico 2}

Freqüência das questões de gráficos por volume e coleção 
Além disso, o gráfico mostra que há um gradual aumento na quantidade de questões envolvendo os tipos de gráficos por volume, principalmente na coleção Vivência e Construção - Matemática, que vai de 5\%, no volume 2, para 58\%, no volume 4.

Com relação aos tipos de gráficos observados nas questões selecionadas sobre interpretação e construção de gráficos, encontramos, nas três coleções, os seguintes: de setores (pizzas), de colunas e/ ou barras, de linha, de barras bidirecionais, de formas geométricas, pictóricos e cartográficos. Entre as atividades de gráficos de barras e/ ou colunas selecionadas realizamos uma subdivisão, que classificamos do seguinte modo: gráficos de barras/ colunas de freqüência, barras/ colunas múltiplas, barras/ colunas múltiplas de freqüência e barras/ colunas compostas.

Q uanto à distribuição desses tipos de gráficos, nas três coleções, encontramos a seguinte divisão:

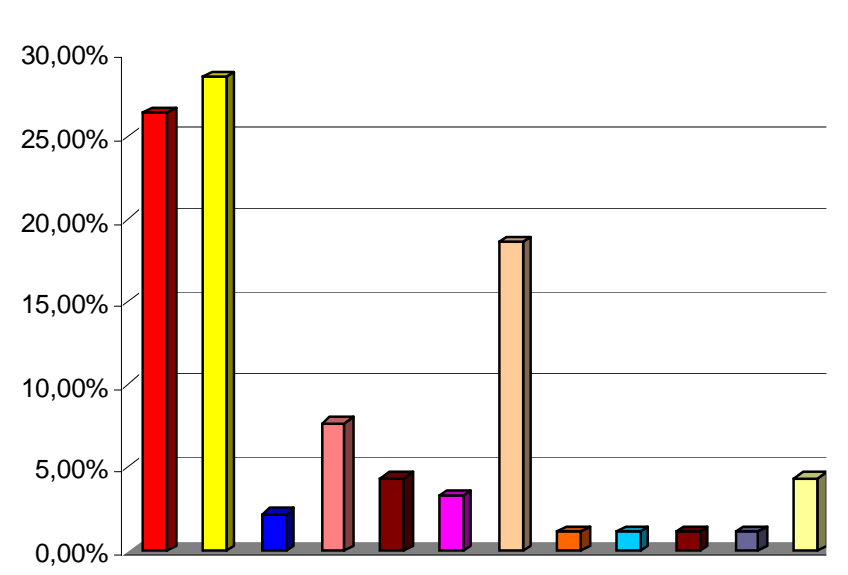

$\square$ setores

$\square$ coluna/barra

口 coluna/barra frequência

$\square$ coluna /barra múltiplas

$\square$ coluna/barra múltiplas frequência

$\square$ barras bidirecionais

$\square$ linha

$\square$ formas geometricas

$\square$ barras compostas

q gráfico pictórico

$\square$ gráficos de pontos

$\square$ gráfico de setores envolvendo porcentagem

\section{Gráfico 3}

Gráfico de freqüência dos tipos de gráficos nas três coleções

Como podemos observar, a maioria das atividades com gráficos apresentada nos livros didáticos é do tipo gráficos de barras e/ ou colunas, mesmo considerando esse tipo dentro das categorias citadas na subdivisão. Além disso, observamos que os gráficos de setores (do tipo pizza) e os gráficos de linhas são bastante trabalhados, ao contrário dos outros tipos, como, por exemplo, os gráficos pictóricos, que representam, apenas, $1 \%$ das questões.

Referindo-nos à análise da comparação entre as coleções, com relação à quantidade de tipos de gráficos explorados em cada livro didático, observamos que a Coleção Matemática com o Sarquis apresentou a maior variedade de gráficos nas questões, como pode ser observado na comparação dos três gráficos a seguir: 


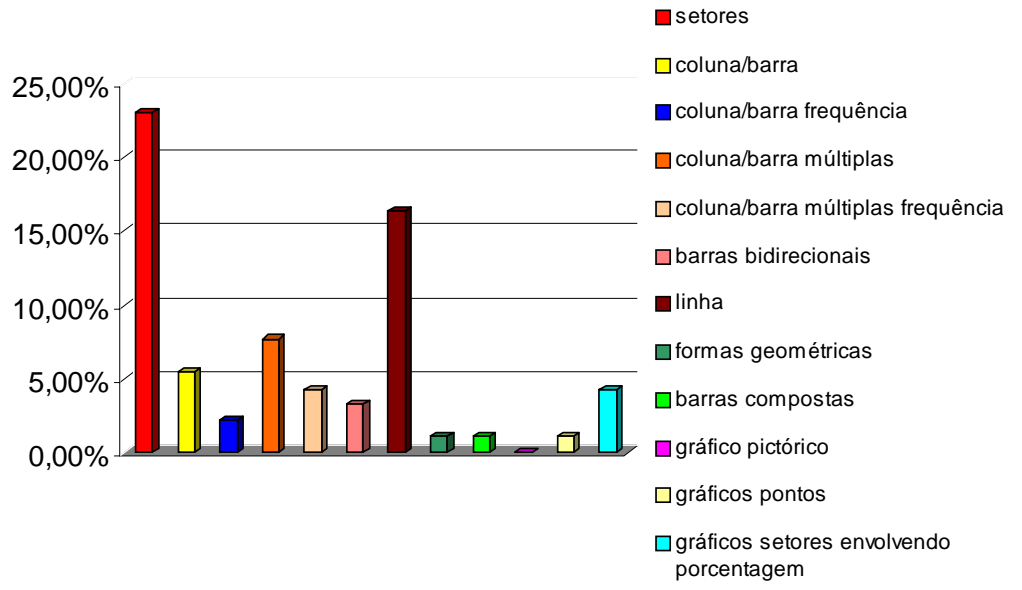

\section{Gráfico 4}

Freqüência de tipos de gráficos na Coleção Matemática com o Sarquis

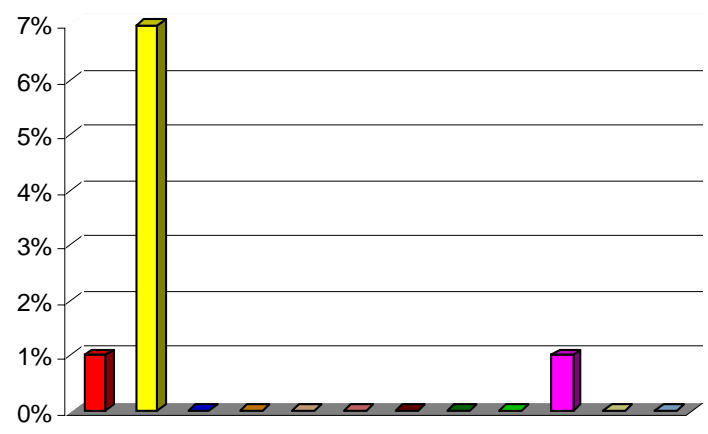

$\square$ setores

$\square$ coluna/barra

$\square$ coluna/barra frequência

$\square$ coluna/barra múltiplas

$\square$ coluna/barra múltiplas frequência

$\square$ barras bidirecionais

$\square$ linha

$\square$ formas geométricas

$\square$ barras compostas

$\square$ gráfico pictórico

$\square$ gráficos pontos

$\square$ gráficos setores envolvendo porcentagem

\section{Gráfico 5}

Gráfico de freqüência de tipos de gráficos na coleção Novo Tempo 


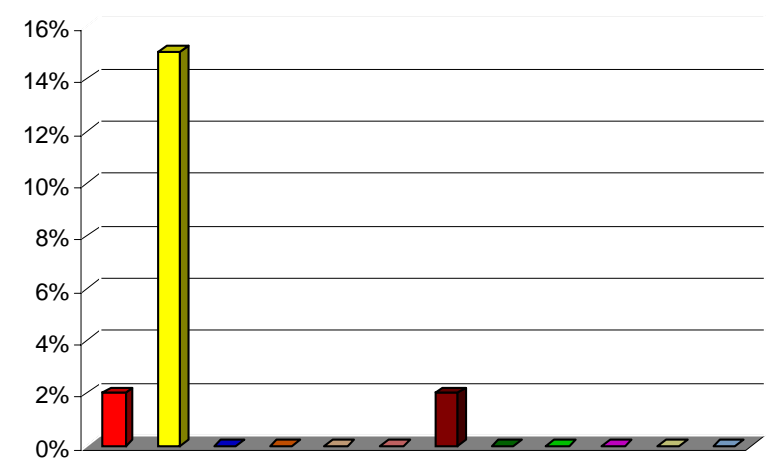

$\square$ setores
$\square$ coluna/barra
$\square$ coluna/barra frequência
$\square$ coluna/barra múltiplas
$\square$ coluna/barra múltiplas frequência
$\square$ barras bidirecionais
$\square$ linha
$\square$ formas geométricas
$\square$ barras compostas
$\square$ gráfico pictórico
$\square$ gráficos pontos
$\square$ gráficos setores envolvendo
porcentagem

\section{Gráfico 6}

Gráfico de freqüência de tipos de gráficos na coleção Vivência e Construção - Matemática

É interessante notar que nas três coleções estão presentes, em maior número, atividades com gráficos de barras e/ ou colunas, os quais também são considerados como os mais utilizados pelos meios de comunicação, como revistas, jornais e televisão (Lima, 1998).

Com relação aos conteúdos explorados por cada atividade, em todas as coleções encontramos as seguintes categorias: localização de pontos (máximo e mínimo) de extrapolação do gráfico; localização do valor da frequiência de uma categoria; localização de uma categoria, a partir do valor da freqüência; cálculo de médias; quantificação de variação (crescimento, decrescimento e estabilidade); soma total de valores; comparação de somas, estimativa, ordenação de valores, medidas de tamanho ou identificação de valor; interpretação, a partir do cálculo da diferença e da soma.

Entretanto, quando contabilizando a quantidade de perguntas sobre gráficos, em cada questão, observamos que a coleção Vivência e Construção - Matemática apresentou o maior número delas por atividade que exploravam as categorias acima citadas, chegando, inclusive, a superar a quantidade de questões da coleção 1(Matemática com Sarquis), o que pode ser observado no gráfico 7:

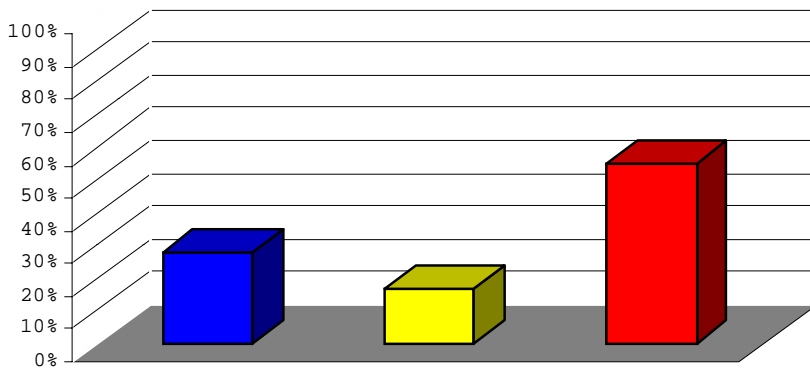

Matemática com o Sarquis

$\square$ Novo Tempo

$\square$ Vivência e Construção

\section{Gráfico 7}

Gráfico de freqüência de questões por coleção 
Este dado é muito interessante, pois mostra que, apesar de a coleção Matemática com o Sarquis apresentar maior quantidade de atividades com tipos de gráficos (70\%), foi a coleção Vivência e Construção - Matemática que teve a maior quantidade de conteúdos explorados pelas questões de gráficos (55\%, contra 28\% obtidos pela coleção 1). Esse resultado ocorreu em virtude das questões relacionadas à construção e interpretação de gráficos apresentadas na coleção 1 explorarem, apenas, de uma a três questões por gráficos, o que não aconteceu nas atividades da coleção 2.

D estacamos, ainda, que a coleção Novo Tempo, no que se refere à quantidade de tipos de gráficos e questões, sempre apresentou quantidade média em comparação com as outras coleções. Isso pode ser observado em todos os gráficos já apresentados.

Com relação à freqüência dos conteúdos explorados por cada atividade contida nas três coleções, observamos que as categorias localização de pontos (máximo), localização de pontos (mínimo), localização do valor da freqüência de uma categoria, soma total de valores, são as mais apresentadas nas atividades, como bem representa o gráfico a seguir:

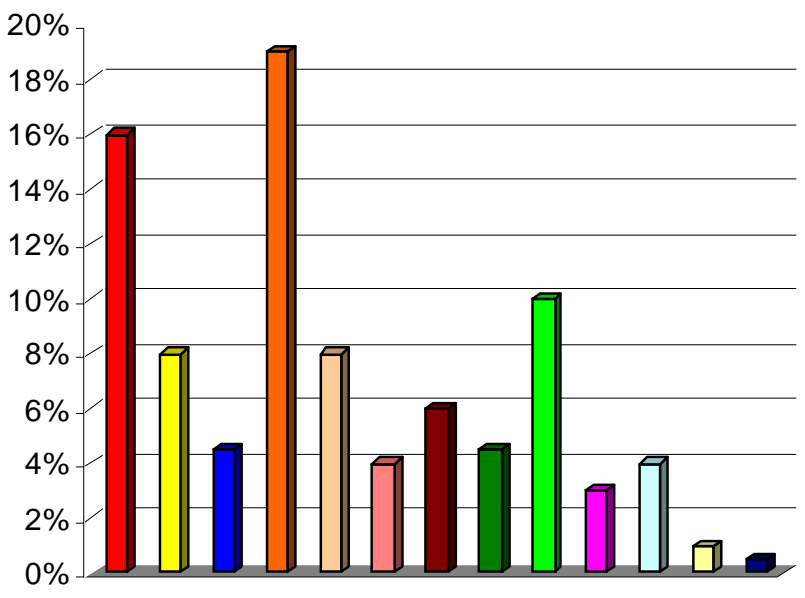

Gráfico 8

Gráfico da quantidade de conteúdos nas atividades das três coleções $\square$ localização de ponto (máximo)

$\square$ localização de ponto (mínimo)

• extrapolação do gráfico

$\square$ localizar o valor da freque. de uma categoria

$\square$ localizar uma categ. A partir da

frequencia

$\square$ avaliar méidas

quantificarvariação (crescimento)

$\square$ quantificar variação (estabilidade)

$\square$ soma total de valores

$\square$ comparar somas

$\square$ construção de gráficos

$\square$ quantificar variação

(decrescimento)

estimativa

A partir da análise da freqüência das questões sobre tratamento da informação distribuída pelos volumes de cada coleção, identificamos que não existiu um padrão gradual na quantidade de questões, por volume, pois, ora um conteúdo era mais explorado no volume 1, ora no volume 2, e assim por diante, como demonstra a tabela a seguir: 
O estudo do tratamento da informação...

Tabela 1

Tabela de freqüência de temas por coleção.

\begin{tabular}{|c|c|c|c|c|c|c|c|c|c|c|c|c|c|}
\hline \multirow[t]{3}{*}{ Conteúdos } & \multicolumn{12}{|c|}{ Coleção/Volume } & \multirow[t]{3}{*}{ Total } \\
\hline & \multicolumn{4}{|c|}{ Coleção 1} & \multicolumn{4}{|c|}{ Coleção 1} & \multicolumn{4}{|c|}{ Coleção 1} & \\
\hline & 1 & 2 & 3 & 4 & 1 & 2 & 3 & 4 & 1 & 2 & 3 & 4 & \\
\hline Localização de pontos (máximo) & & 2 & 2 & 2 & & 2 & 1 & & & 4 & 6 & 9 & 28 \\
\hline Localização de pontos (mínimo) & & 2 & & & & & & & & 3 & 5 & 4 & 14 \\
\hline Extrapolação de gráfico & & 1 & 2 & & & & 3 & 1 & & & & 1 & 8 \\
\hline $\begin{array}{l}\text { Localizar o valor da freqüência de } \\
\text { uma categoria }\end{array}$ & & 1 & 3 & 2 & & 4 & & 4 & & 2 & 14 & 3 & 33 \\
\hline $\begin{array}{l}\text { Localizar uma categoria a partir da } \\
\text { freqüência }\end{array}$ & & & & 1 & & 1 & & & & 1 & 5 & 7 & 15 \\
\hline Avaliar média & & & & & & & & 3 & & & & 4 & 7 \\
\hline Quantificar variação (crescimento) & & 2 & 1 & 1 & & & & & & & 4 & 3 & 11 \\
\hline Quantificar variação (estabilidade) & & 2 & 1 & 1 & & & & & & 1 & 4 & 3 & 12 \\
\hline Soma total de valores & & & 1 & 2 & & 1 & 1 & 2 & & & 5 & 5 & 17 \\
\hline Comparar somas & & & 4 & 1 & & & & & & & & & 5 \\
\hline Construção de gráficos & & & 1 & 1 & & 1 & 1 & 2 & & 1 & & & 7 \\
\hline $\begin{array}{l}\text { Quantificar variação } \\
\text { (decrescimento) }\end{array}$ & & & & 1 & & & & & & 1 & & & 2 \\
\hline Estimativa & & & 1 & & & & & & & & & & 1 \\
\hline
\end{tabular}

A tabela reforça os dados apresentados no gráfico 8, que demonstra o percentual da quantidade de conteúdos explorados nas três coleções.

\section{Considerações finais}

A análise dos livros didáticos de Matemática de 1aa a $4^{\underline{a}}$ série recomendados com distinção pelo PNLD (BRASIL, 2000), mostrou que o trabalho com o tratamento da informação está distante do ideal. Isso pôde ser observado mediante os dados analisados, nos quais apenas a coleção 1 apresentou 0 trabalho com interpretação e construção de gráficos em todos os livros didáticos em seus volumes 2, 3 e 4.

Nas outras coleções, o trabalho de tratamento da informação restringiu-se ao capítulo de estatística e probabilidade, sem atividades relacionadas com outras áreas do conhecimento e não favorecendo, assim, a interdisciplinaridade. Vale ressaltar, ainda, que nenhuma coleção apresentou atividades com tratamento da informação no volume 1.

Em relação aos gráficos apresentados nas coleções, observamos que em todas a maioria é do tipo de barras e colunas. Existe, também, o uso de gráficos de setores (pizza) e de linhas, mas, quando comparamos a freqüência desses tipos nas questões com gráficos, nas três cole- 
ções (gráficos 4, 5 e 6), percebemos claramente o predomínio dos gráficos de barras e de colunas em relação aos outros tipos, principalmente nas coleções 2 e 3.

Ressaltamos, ainda, que a coleção 1 é a que apresenta a maior quantidade de questões com gráficos (64 ou 70\%) em relação às coleções 2 (8 ou 9\%) e 3 (19 ou 20\%). Ela apresentou, também, a maior variedade de tipos de gráficos, tanto de setores, de linha, pictóricos, de pontos etc. como de barras e de colunas, dentro da sub-divisão que realizamos.

Entretanto, em relação à quantidade de perguntas que acompanham os gráficos, observamos que a coleção 3 foi a que apresentou a maior quantidade de perguntas por atividade com gráficos (55\%) em relação às coleções 1 (28\%) e 2 (17\%).

D e modo geral, podemos dizer que, embora as coleções apresentem atividades voltadas à interpretação e construção de gráficos, a maioria não explora os diversos tipos de gráficos e conteúdos por questão, como representado na Tabela 1 e nos gráficos 4 , 5 e 6 . Isso dificulta a apresentação e resolução, por parte dos alunos, de atividades e situações-problema com variados tipos de gráficos.

Com relação aos conteúdos explorados em cada questão distribuída pelas três coleções, observamos que não existiu um padrão para a distribuição dos conteúdos por volume, pois ora um conteúdo era mais trabalhado no volume 2 , ora no volume 3 , e assim por diante. Além disso, observamos também que os conteúdos mais explorados pelas questões contidas nas três coleções foram: localização do valor da freqüência de uma categoria, localização de pontos máximos, localização de pontos mínimos, soma total de valores, localização de uma categoria a partir do valor da freqüência.

Esses dados são muito interessantes, pois percebemos em algumas pesquisas (Santos e Gitirana, 1999; G uimarães, 2002; Magina et al., 1997; Bell e Janvier, 1981) que os conteúdos referentes à localização de pontos (máximo e mínimo) são considerados como os mais fáceis e utilizados pelas crianças como estratégia de resolução das questões de interpretação de gráficos.

Esses resultados mostram que o trabalho com o tratamento da informação realizado pelos livros didáticos encontra-se distante de possibilitar aos alunos a construção de procedimentos para coletar, organizar, comunicar e interpretar dados, utilizando tabelas, gráficos e representações que aparecem freqüentemente em seu dia-a-dia. Esta afirmação se deve ao fato de que, em sua maioria, as atividades observadas nos livros didáticos analisados não propõem situações em que os alunos sejam incentivados a interpretar diferentes tipos de gráficos e a resolver questões com diversos tipos de conteúdos estatísticos e matemáticos, como apresentado na Tabela 1.

O utro ponto considerado um dado importante e que deve ser revisto por autores de livros didáticos refere-se ao desenvolvimento de um trabalho contínuo com interpretação de gráficos em todo o livro didático, e não apenas em um capítulo. Além disso, ele deve ser articulado a outros conteúdos, utilizando diversas representações gráficas, o que não foi observado, pois a maioria dos gráficos encontrados nos livros didáticos foi de barras e de colunas.

Para finalizar, é importante ressaltar que não estamos desmerecendo o trabalho com o tratamento da informação apresentada nos livros didáticos e, sim, chamando a atenção para o tipo de trabalho que está sendo proposto por esses instrumentos. Estamos, também, refletindo sobre a necessidade de que se desenvolvam atividades que favoreçam a interpretação de 
O estudo do tratamento da informação...

diferentes tipos de gráficos, além de proporcionar uma maior variedade de questões e atividades que trabalhem com diversos conteúdos estatísticos e matemáticos, principalmente aqueles que utilizam fenômenos do cotidiano dos alunos.

\section{Referências}

ALBUQUERQ UE, E.B.C. 0 discurso dos professores sobre a utilização do livro didático: 0 que eles afirmam/ negam em relação a este material? Recife, 2002 (mimeo).

BELL, A.; JANVIER, C. The interpretation of graphs representing situations. For learning of Mathematics, v. 2, p. 34-42, 1981.

BRASIL. Ministério da Educação e D esporto - Secretaria do Ensino Fundamental. Parâmetros Curriculares Nacionais: Matemática. Brasília: MEC/ SEF, 1997.

. Ministério da Educação e do Desporto. Guia de livros didáticos: 1ํa a $4^{\mathrm{a}}$ séries. Brasília: MEC, 2004.

CARRAHER, D.; SCHLIEMANN, A.; NEMIROVSKY, R. Understanding graphs without schooling. Hands on! Cambrigge: TERC, 1995.

GUIMARÃE S, G. L. Interpretando e construindo gráficos de barras. Recife. 2002. 258p. Tese (D outorado em Psicologia Cognitiva) - Universidade Federal de Pernambuco.

LAJO LO, M. Livro didático e qualidade de ensino. In: E m Aberto. Ministério da Educação e Desporto SEDIAE/ INEP, v. 16, n. 69, 1996.

LIMA, L. M. T. Interpretação de gráficos de quantidades veiculados pela mídia imprensa: um estudo exploratónio. Recife. 1998. Dissertação (Mestrado em Psicologia Cognitiva) - Departamento de Psicologia da Universidade Federal de Pernambuco.

MAGINA, S. M. P.; G ITIRANA, V.; Maranhão, M. C. S. A. Interpretação de gráficos e diagramas em ambiente computacional de manipulação de dados. Projeto de Pesquisa financiado pelo CNPq. São Paulo, 1997. (não publicado).

MEIRA, L. Making sense of instructional devices: the emergence of transparency in mathematical activity. Journal for Resarch in Mathematics Education, v. 29, n. 2, p. 121-142, 1998.

Gráficos de quantidades na vida diária e na mídia impressa. Recife: Departamento de Psicologia/ UFPE, 1998. 
NATIONAl COUNCIL OF TEACHERS OF MATHEMATICS. NCTM. Principles and standards for school Mathematics. USA: Reston, 2000.

SANTOS, M. S.; G ITIRANA, V. A interpretação de gráficos de barra, com variáveis numéricas, em um ambiente computacional de manipulação de dados. In: ENCONTRO DE PESQUISA EDUCACIO NAL DO NORDESTE, 14., 1999, Salvador. Anais... Salvador: EPEN, 1999.

SILVA, N. P. Estatística auto-explicativa. São Paulo: Érica, 1998.

SILVA, E. T. Livro didático: do ritual de passagem à ultrapassagem. In: Em Aberto. Ministério da Educação e Desporto SEDIAE/ INEP, v. 16, n. 69, 1996.

TO LED O, G. L. Estatística básica. São Paulo: Atlas, 1985. 